\title{
Logros de aprendizaje en comprensión lectora y matemática en el Perú
}

\section{Learning gains in reading comprehension and math in Perú}

Idel Vexler ${ }^{1}$

Universidad de San Martín de Porres

\section{INTRODUCCIÓN}

En el trabajo educativo uno de los temas más complejos y controversiales es el referido a la evaluación de los aprendizajes, y es que no solamente tiene un carácter educativo y académico, sino que muchas veces sus resultados no son manejados convenientemente para tomar decisiones de mejoramiento y afianzamiento educativo. Generalmente son utilizados -desde posiciones político-partidarias- para generar un clima de descalificación y desconfianza en los sistemas educativos.

La evaluación de la calidad educativa que se evidencia por logros de aprendizajes significativos puede tener una dimensión macro a nivel institucional, local, regional, nacional e internacional. El Perú actualmente interviene en las pruebas nacionales e internacionales de la UNESCO y PISA. También existe una dimensión micro o especifica que tiene su máxima expresión en los procesos particulares de evaluación a cada niño, niña, adolescente y adulto en un marco de una consistente y permanente formación integral.

Por otro lado, debe quedar claro que hay otros tipos de evaluaciones de la calidad educativa, como las referidas a la acreditación de las instituciones educativas, así como las de certificación y recertificación de las competencias profesionales de las personas.

Las evaluaciones nacionales son realizadas desde

1994. Estas se han aplicado a niños de primaria mediante normas y criterios o estándares. En el 2000 y 2004 se aplicaron pruebas nacionales a estudiantes de primaria y secundaria; sin embargo, desde el 2007 hasta la actualidad se viene aplicando las evaluaciones censales de niños y niñas de segundo grado (que tienen como lengua materna el castellano) y cuarto grado (Escuelas Interculturales Bilingües) del nivel de Educación Primaria.

El 4 y 5 de diciembre de 2012 fue realizada la Evaluación Censal de Estudiantes (ECE 2012). Abarcó el $97,7 \%$ de instituciones educativas y se aplicó al $89,4 \%$ de los niños y niñas de segundo grado de primaria (en comprensión lectora y Matemática), así como a los de

1 Educador; especialista en gestión pedagógica, currículo y evaluación; viceministro de Educación; consultor y asesor educativo a nivel nacional e internacional; articulista de los diarios Correo y La República. Profesor titular del Instituto de Gobierno y de Gestión Pública de la Universidad de San Martín de Porres. Miembro del Consejo Nacional de Educación y presidente de la Comisión de Educación de la Cámara de Comercio de Lima. 
cuarto grado de primaria (en comprensión lectora) que realizan estudios en contextos interculturales bilingües (1). Sus resultados fueron dados a conocer por la ministra de Educación, Patricia Salas O’Brien, el martes 2 de abril de 2013. En este texto nos referimos a los resultados de la ECE 2012, luego a precisiones sobre la evolución de los niveles de logro de las evaluaciones 2007 al 2012, y por último, planteamos algunas conclusiones y reflexiones.



Tabla $N^{\circ}$ 2: Logros en zona rural en Matemática 2010-2012

\begin{tabular}{llll}
\hline Rural & $5,8 \%$ & $3,7 \%$ & $4,1 \%$ \\
Diferencias $\%$ & & $-2,1 \%$ & $0,4 \%$ \\
Año de censo (ECE) & $\mathbf{2 0 1 0}$ & $\mathbf{2 0 1 1}$ & $\mathbf{2 0 1 2}$ \\
\hline
\end{tabular}

\section{Resultados ECE 2012}

De acuerdo con la Evaluación Censal de Estudiantes 2012 (1), el 30,9\% de los alumnos tiene logros satisfactorios en comprensión lectora (CL) y el 12,8 en Matemática $(M)$, pero ambos resultados aún están lejos del $33 \%$ y 15,7 \% que como metas fueron previstas alcanzar en las respectivas áreas en el 2012, según el Plan Educativo Sectorial Multianual 2012$2016(2)$.

En las zonas rurales el $7 \%$ obtiene logros satisfactorios en comprensión lectora aumentando 1,1 \% en comparación con el 2011 ; y en Matemática obtiene el 4,1\%, con un aumento de 0,4\% en comparación también con el 2011 (tablas $N^{\circ} 1$ y $N^{\circ} 2$ ).

En las tablas $\mathrm{N}^{\circ} 3$ y $\mathrm{N}^{\circ} 4$, entre las escuelas privadas y públicas se puede observar, en Comprensión Lectora del 2011 al 2012, una brecha de $+0,1 \%$, y en Matemática una brecha de $-2,7 \%$. La disminución en
Tabla $N^{\circ} 3$ : Brechas entre escuelas privadas $y$ públicas en CL 2010-2012

\begin{tabular}{lccc}
\multicolumn{5}{c}{ públicas en CL 2010-2012 } \\
\hline \multicolumn{5}{c}{$\begin{array}{c}\text { Comprensión Lectora logros } \\
\text { satisfactorios 2010-2012 }\end{array}$} \\
\hline Privada & $48,6 \%$ & $50,3 \%$ & $51,4 \%$ \\
Pública & $22,8 \%$ & $23,0 \%$ & $24,0 \%$ \\
Brechas \% & $25,8 \%$ & $27,3 \%$ & $27,4 \%$ \\
Año de censo (ECE) & $\mathbf{2 0 1 0}$ & 2017 & $\mathbf{2 0 1 2}$ \\
\hline
\end{tabular}

Tabla $N^{\circ}$ 4: Brechas entre escuelas privadas $y$ públicas en Matemática 2010-2012

Matemática logros satisfactorios 2010-2012

\begin{tabular}{lccc}
\hline Privada & $20,9 \%$ & $18,9 \%$ & $16,5 \%$ \\
Pública & $11,7 \%$ & $11,3 \%$ & $115 \%$ \\
Brechas \% & $9,2 \%$ & $7,6 \%$ & $4,9 \%$ \\
Año de censo (ECE) & 2010 & 2017 & 2012 \\
\hline
\end{tabular}

la diferencia en Matemática se debe a que las escuelas privadas han tenido un decrecimiento de $2,4 \%$.

En las tablas $\mathrm{N}^{\circ} 5$ y $\mathrm{N}^{\circ} 6$ podemos notar que entre las escuelas urbanas y rurales en comprensión lectora del 2011 al 2012 existe una brecha de $+0,1 \%$, y en Matemática una brecha de $-1,1 \%$. En Matemática la disminución en la brecha entre educación rural y urbana se debe a que esta última ha tenido un decrecimiento de $0,6 \% ; 15,8 \%$ (2011) a 15,2\% (2012).

Tabla $N^{\circ}$ 5: Brechas entre escuelas urbanas $y$ rurales en $C L$ 2010-2012

\begin{tabular}{lrrr}
\hline \multicolumn{4}{c}{$\begin{array}{c}\text { Comprensión Lectora logros } \\
\text { satisfactorios 2010 - 2012 }\end{array}$} \\
\hline Urbana & $35,5 \%$ & $36,3 \%$ & $37,5 \%$ \\
Rural & $7,6 \%$ & $5,9 \%$ & $7,0 \%$ \\
Brechas \% & $27,9 \%$ & $30,4 \%$ & $30,5 \%$ \\
Año de censo (ECE) & 2010 & 2017 & $\mathbf{2 0 1 2}$
\end{tabular}

Tabla $N^{\circ}$ 6: Brechas entre escuelas urbanas $y$ rurales en Matemática 2010-2012

Matemática logros satisfactorios $2010-2012$

\begin{tabular}{lrrr}
\hline Urbana & $16,4 \%$ & $15,8 \%$ & $15,2 \%$ \\
Rural & $5,8 \%$ & $3,7 \%$ & $-11 \%$ \\
Brechas \% & $10,6 \%$ & $12,1 \%$ & $11,0 \%$ \\
Año de censo (ECE) & 2010 & 2017 & $\mathbf{2 0 1 2}$
\end{tabular}






Figura $N^{\circ}$ 1: Comparación de resultados satisfactorios alcanzados en comprensión lectora por las escuelas privadas y públicas de los distritos de Lima Metropolitana.



Figura $\mathrm{N}^{\circ}$ 2: Comparación de resultados satisfactorios alcanzados en Matemática por las escuelas privadas y públicas de los distritos de Lima Metropolitana.

De lo señalado se puede mencionar que las brechas se mantienen casi iguales. No hay mayores cambios en los aprendizajes en los alumnos de las áreas rurales e interculturales bilingües. En los años 2011 y 2012 se ha dispuesto de un presupuesto elevado de aproximadamente dos mil millones de soles, a diferencia de años anteriores.

Por otro lado, en los distritos populares de Lima se observa que los resultados en comprensión lectora de la escuela privada son ligeramente mayores que los de la escuela pública; sin embargo, se puede 


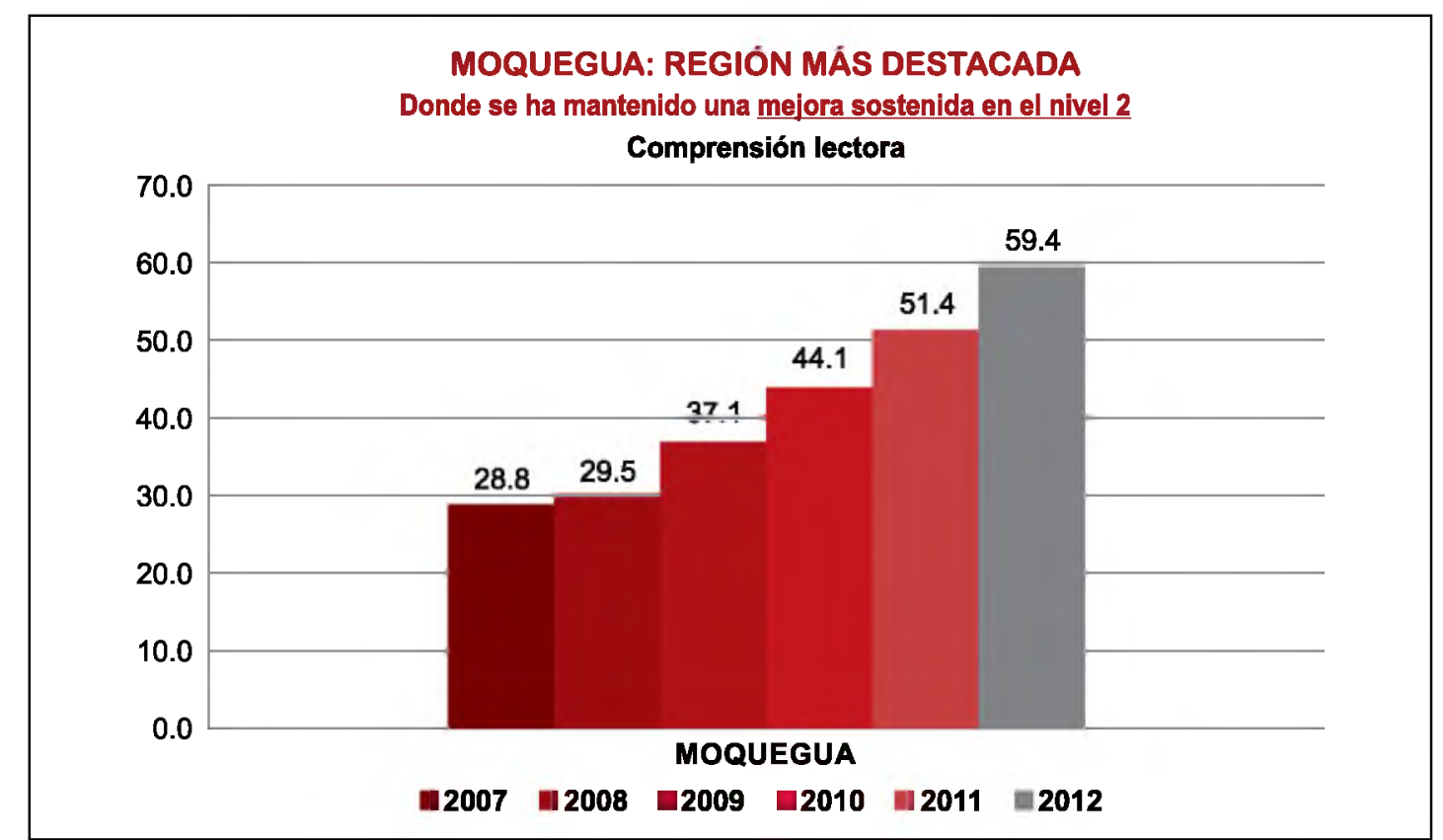

Figura $\mathrm{N}^{\circ}$ 3: El mayor resultado nacional obtenido por Moquegua en comprensión lectora y la más destacada evolución de mejora sostenida en el nivel satisfactorio que como región tiene en esta área (ECE: 2007-2012)

afirmar que son similares (figura $\mathrm{N}^{0} 1$ ).

En estos mismos ámbitos, los resultados de la escuela pública en Matemática son mejores que los de la escuela privada, lo cual se viene apreciando desde el 2011 (figura $N^{\circ} 2$ ). No obstante, hay que tener cuidado de hacer creer a la comunidad educativa que las escuelas públicas tienen buena calidad en Matemática, lo cual no es así.

Esto debe llamar mucho la atención del Ministerio de Educación y del INDECOPI, pues se debe exigir a las escuelas privadas, de estas jurisdicciones, mejorar urgentemente sus logros de aprendizaje. La Dirección Regional y UGEL de Lima debieran asumir la responsabilidad que les compete por autorizar su funcionamiento sin realizar un riguroso control de calidad.

Asimismo, los resultados indican que las regiones de Moquegua y Tacna son las que mejores logros han tenido en comprensión lectora y Matemática. Las regiones que muestran una mejora sostenida en comprensión lectora, desde el 2007 hasta el 2012, son Moquegua (figura $N^{\circ} 3$ ), Lima Metropolitana, Callao, Amazonas y Madre de Dios.

Lo preocupante es que, entre otras regiones, la que no avanza y tiene muy pobres resultados en Comprensión Lectora y Matemática, es Loreto (tablas $\mathrm{N}^{\circ} 7$ y $\mathrm{N}^{\circ} 8$ ), que continúa en el último lugar (1).

\section{Evolución de los logros de aprendizaje del 2007 al 2012}

Ahora es conveniente mirar la evolución de los niveles de aprendizaje en los últimos seis años. En Comprensión Lectora, el 15,9\% de alumnos evaluados el 2007 alcanzaron el nivel satisfactorio; el 2008 lograron este nivel el 16,9\%; el 2009, el 23,1\%; el 2010 , el $28,7 \%$; el 2011 , el $29,8 \%$; y el 2012 , el

Tabla $N^{\circ}$ 7: Niveles de logro de la región Loreto en comprensión Lectora (ECE: 20102012)

\begin{tabular}{lccc}
\hline \multicolumn{4}{c}{ Loreto: Comprensión Lectora } \\
& 2010 & 2011 & 2012 \\
\hline Nivel 2: Satisfactorio & $5,0 \%$ & $6,1 \%$ & $6,3 \%$ \\
Nivel 1: En proceso & $30,3 \%$ & $30,7 \%$ & $35,7 \%$ \\
Debajo del nivel 1: & $64,6 \%$ & $63,2 \%$ & $58,0 \%$ \\
En inicio & & & \\
\end{tabular}




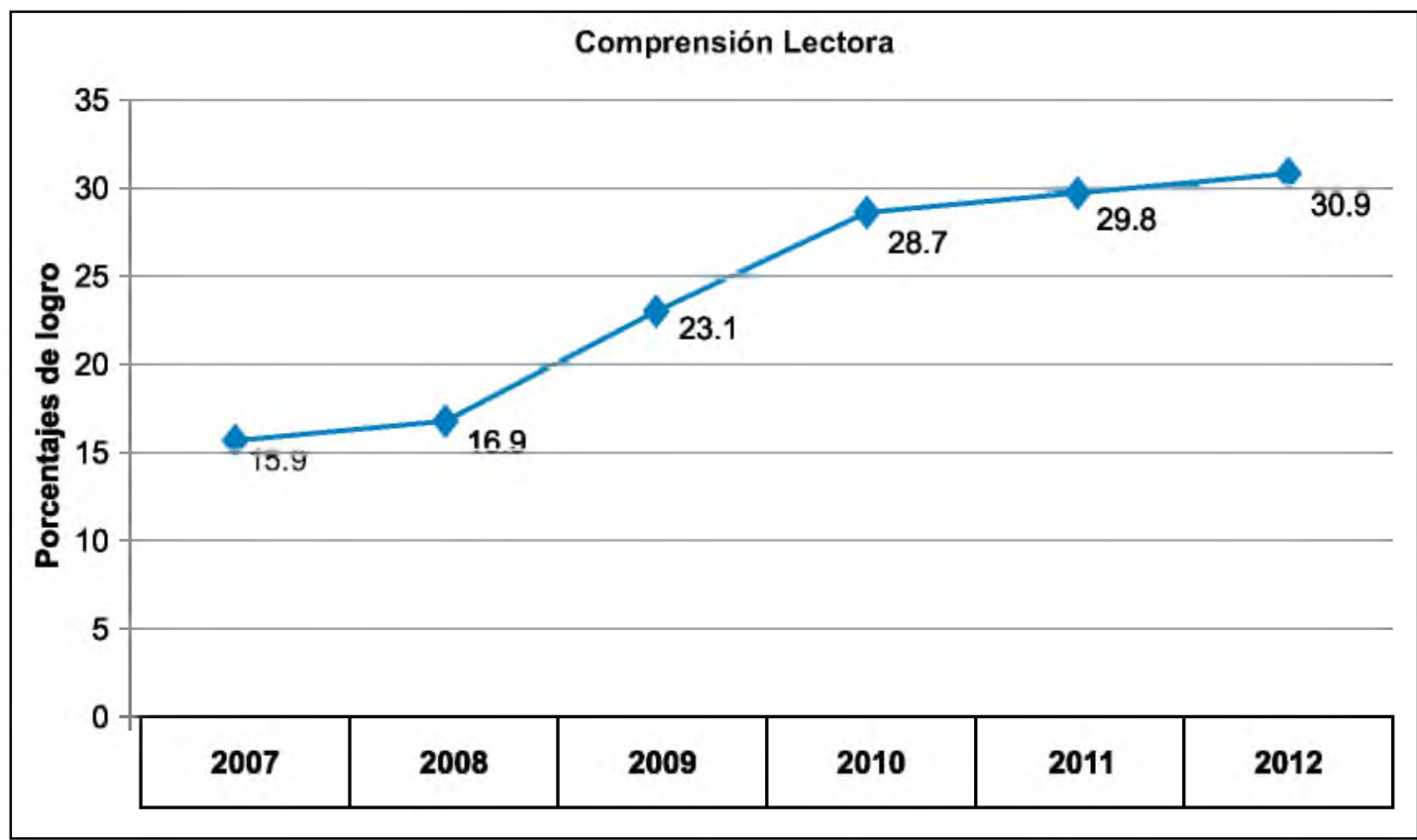

Figura $N^{\circ}$ 4: Evolución de logro de nivel satisfactorio en Comprensión Lectora (ECE: 2007-2012).

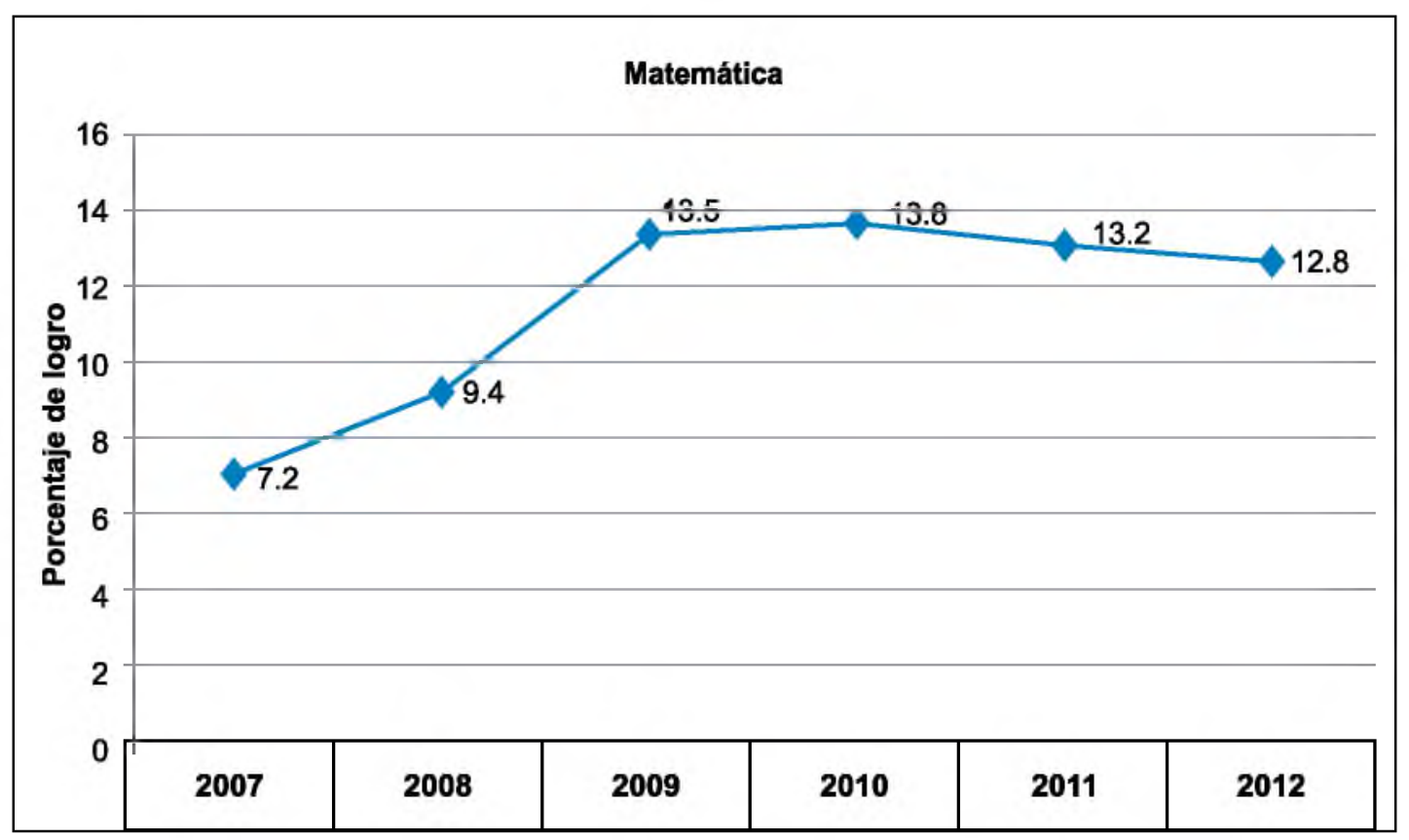

Figura N5: Evolución de logro de nivel satisfactorio en Matemática (ECE: 2007-2012).

$30,9 \%(3)$. Esto muestra que entre el 2007 $y$ el 2010 hubo un incremento sostenido $y$ claro de $12,8 \%$ de alumnos que obtuvieron el más alto nivel de logro. Pero, en los años 2011 y 2012 el incremento fue de solo $2,2 \%$. (figura $\mathrm{N}^{\circ} 4$ ).

En Matemática, el 7,2 \% de alumnos evaluados el 2007 obtuvieron el nivel satisfactorio; el 2008 lograron este nivel el 9,4\%; el 2009, el 13,5\%; el 2010, el 13,8 $\%$; el 2011, el 13,2\%; y el 2012, el 12,8 $\%$ (3). Entre el 2007 y el 2010 hubo un incremento sostenido de 6,6\% de alumnos que alcanzaron el más alto nivel de logro.

En los años 2011 y 2012, sin embargo, disminuyó $1 \%$. (figura $\mathrm{N}^{\circ} 5$ ). 
Tabla N ${ }^{\circ}$ 8: Niveles de logro de la región Loreto en Matemática (ECE: 2010-2012)

\begin{tabular}{lrrr}
\hline \multicolumn{4}{c}{ Loreto: Matemática } \\
& 2010 & 2011 & 2012 \\
\hline Nivel 2: Satisfactorio & $1,0 \%$ & $1,4 \%$ & $1,4 \%$ \\
Nivel 1: En proceso & $10,7 \%$ & $9,9 \%$ & $11,5 \%$ \\
Debajo del nivel 1: & $88,2 \%$ & $88,7 \%$ & $87,1 \%$ \\
En inicio & & & \\
\end{tabular}

\section{CONCLUSIONES}

A pesar de que las autoridades actuales del Ministerio de Educación tienen como alta prioridad disminuir las brechas, éstas se mantienen casi intactas. Además, se evidencia que para mejorar los aprendizajes en las áreas rurales y ámbitos interculturales bilingües, en los años 2011 y 2012 se ha dispuesto de un presupuesto elevado de alrededor de dos mil millones de soles, a diferencia de años anteriores.

Las regiones de Moquegua y Tacna son las que mejores logros han tenido en comprensión lectora y Matemática.

Las regiones que muestran una mejora sostenida en comprensión lectora, desde el 2007 hasta el 2012, son Moquegua, Lima Metropolitana, Callao, Amazonas y Madre de Dios.

En logros de aprendizaje de comprensión lectora y Matemática entre el 2007 y el 2010 hubo avances muy significativos, de manera sostenida e importante en comprensión lectora, aunque de manera precaria aún en Matemática.

Entre los años 2011 y 2012 no estamos mejor, ni peor, sino estancados

Como reflexiones finales podemos decir que corresponde manejar estos resultados con responsabilidad, sin asumir una postura exitista ni derrotista. Claro está que las autoridades actuales deben reconocer que a 20 meses de gestión no hay mejora de la calidad educativa. $Y$ hay que desarrollar, por cierto, con el apoyo de todos los actores educativos, medidas efectivas de mejoramiento pedagógico. No olvidemos que las metas al 2016 son: en comprensión lectora 55\% y en Matemática $35 \%$ (2). Los resultados de las evaluaciones, siempre, deben ser utilizados para mejorar, ese es el reto. Pasemos del discurso a las realizaciones, para ello hay que priorizar el trabajo en las escuelas y aulas desarrollando competencias en el marco de la diversidad nacional y la sociedad global del conocimiento. Ese es el gran desafío para avanzar hacia el mejoramiento de la calidad educativa de la escuela peruana.

\section{REFERENCIAS BIBLIOGRÁFICAS}

1. Ministerio de Educación. Resultados de la Evaluación Censal de Estudiantes 2012 (ECE 2012) [Internet]. Lima: Unidad de Medición de la Calidad Educativa (UMC), Ministerio de Educación; 2013. Disponible en: http://umc.minedu.gob. $p e / ? p=1405$

2. Ministerio de Educación. Plan Estratégico Sectorial Multianual de Educación (PESEM) 2012-2016 [Internet]. Lima: Ministerio de Educación; 2012 [79 páginas]. Disponible en: http:// www.minedu.gob.pe/normatividad/ planesoperativos.php

3. Ministerio de Educación. Resultados generales de la Evaluación Censal de Estudiantes (ECE) de segundo grado de primaria, período 2007-2012. Lima: Unidad de Medición de la Calidad Educativa (UMC), Ministerio de Educación; 2013 [2 pantallas]. Disponible en: http://umc.minedu.gob. $\mathrm{pe} / \mathrm{p}=1357$. 